Journal for ... ImmunoTherapy of Cancer

\section{Pan-cancer analysis of CD274 (PD-L1) mutations in 314,631 patient samples and subset correlation with PD-L1 protein expression}

To cite: Huang RS.P, Decker B, Murugesan $\mathrm{K}$, et al. Pan-cancer analysis of CD274 (PD-L1) mutations in 314,631 patient samples and subset correlation with PD-L1 protein expression. Journal for ImmunoTherapy of Cancer 2021;9:e002558. doi:10.1136/jitc-2021-002558

- Additional supplemental material is published online only. To view, please visit the journal online (http://dx.doi.org/10. 1136/jitc-2021-002558).

Accepted 10 May 2021

Check for updates

(C) Author(s) (or their employer(s)) 2021. Re-use permitted under CC BY-NC. No commercial re-use. See rights and permissions. Published by BMJ.

${ }^{1}$ Foundation Medicine Inc, Cambridge, Massachusetts, USA ${ }^{2}$ Wake Forest Comprehensive Cancer Center, Winston-Salem, North Carolina, USA

${ }^{3}$ Department of Pathology, Wake Forest School of Medicine, Winston-Salem, North Carolina, USA

${ }^{4}$ Department of Pathology, State University of New York (SUNY) Upstate Medical University, Syracuse, New York, USA

Correspondence to Dr Richard S.P. Huang; rhuang@foundationmedicine. com

\section{ABSTRACT}

Background The effects of non-amplification short variant (SV) mutations in CD274 (programmed deathligand 1 (PD-L1)) on PD-L1 protein expression and immune checkpoint inhibitors (ICPIs) therapy are unknown. Here, we present a retrospective analysis of $C D 274$ mutations detected by comprehensive genomic profiling (CGP) and correlate these results with tumor-cell PD-L1 immunohistochemistry ( $\mathrm{IHC}$ )-based expression assessment to better understand the relationship between mutations and protein expression of PD-L1.

Methods CGP was performed on hybridization-captured, adaptor ligation-based libraries using DNA and/or RNA extracted from 314,631 tumor samples that were sequenced for up to 406 cancer-related genes and select gene rearrangements. PD-L1 IHC was performed on a subset of cases $(n=58,341)$ using the DAKO 22C3 PD-L1 $\mathrm{IHC}$ assay and scored with the tumor proportion score (TPS).

Results Overall, the prevalence of CD274 SV mutations was low $(0.3 \%, 1081 / 314,631)$ with 577 unique variants. The most common CD274 SV mutations were R260H $(n=51), R 260 C(n=18), R 125 Q(n=12), C 272 f s^{*} 13(n=11)$, R86W ( $n=10)$, and $R 113 H(n=10)$. The prevalence of CD274 mutations varied depending on tumor type with diffuse large B-cell lymphoma $(1.9 \%, 19 / 997)$, cutaneous squamous cell carcinoma $(1.6 \%, 14 / 868)$, endometrial adenocarcinoma $(1.0 \%, 36 / 3740)$, unknown primary melanoma $(0.9 \%, 33 / 3679)$, and cutaneous melanoma $(0.8 \%, 32 / 3874)$ having the highest frequency of mutations. Of the R260H cases concurrently tested with PD-L1 IHC, most $(81.8 \%, 9 / 11)$ had no PD-L1 expression, which contrasts to the five E237K cases where most $(80 \%, 4 / 5)$ had PD-L1 expression. In addition, we saw a significantly lower level of PD-L1 expression in samples with a clonal truncating variant (nonsense or frameshift indel) when compared with samples with a subclonal truncating variants (mean: TPS $=1$ vs TPS $=38 ; p<0.001$ ), and also in clonal versus subclonal missense mutations (mean: TPS $=11$ vs TPS=22, respectively; $p=0.049$ ) Conclusions We defined the landscape of CD274 mutations in a large cohort of tumor types that can be used as a reference for examining CD274 mutations as potential resistance biomarkers for ICPI. Furthermore, we presented novel data on the correlation of $C D 274$ mutations and PD-L1 protein expression, providing important new information on the potential functionality of these mutations and can serve as a basis for future research.

\section{INTRODUCTION}

Immune checkpoint inhibitors (ICPIs) that block the programmed death-ligand 1 and programmed cell death protein 1 (PD-L1/ PD-1) axis have shown great clinical utility in a wide variety of solid tumors and hematologic malignancies. ${ }^{1-4}$ Multiple companion diagnostics (CDx) for ICPI have been developed and subsequently approved by the United States Food and Drug Administration. ${ }^{5}$ A frequent ICPI CDx utilized for multiple tumor types is PD-L1 immunohistochemistry (IHC) which can detect PD-L1 protein expression/ overexpression on tumor cells and tumorinfiltrating immune cells. Multiple clinical trials have shown that in specific tumor types, a certain level of PD-L1 protein expression is necessary in the tumor microenvironment for a PD-L1/PD-1 inhibitor to be efficacious. ${ }^{48}$

The PD-L1 protein is encoded by the approximately $17.6 \quad \mathrm{~kb} \quad \mathrm{CD} 274 \quad$ (PD-L1) gene located on chromosome 9p24.1. ${ }^{9}$ The CD274 Matched Annotation from NCBI and EMBL-EBI transcript (ENST00000381577.4) encodes for a type 1 transmembrane protein that is 290 amino acids long and has immunoglobulin V-like and C-like domains. ${ }^{10}$ Currently, in large public genomic databases like COSMIC, only 229 CD274 nonamplification short variant (SV)-mutated samples have been reported. ${ }^{11} 12$

Previously, two large studies examined PD-L1 protein expression in a variety of tumor types; however, the published literature contains limited data on $C D 274$ SV mutations. ${ }^{1314}$ Here, we present the landscape of 
CD274 SV mutations detected by comprehensive genomic profiling (CGP) in a large pan-cancer genomic database. In addition, for a subset of these SV mutations, we evaluated PD-L1 protein expression via IHC to better understand the effect of these mutations on protein expression of PD-L1.

\section{MATERIALS AND METHODS \\ Sample cohort}

We analyzed all cases that underwent CGP testing at Foundation Medicine, Inc between January 2014 and August 2020. Formalin-fixed, paraffin-embedded (FFPE) tissue of either whole section samples, biopsies, or cytology specimens were received as paraffin blocks or unstained slides from outside institutions during routine clinical care. A board-certified pathologist assigned a diagnosis for each specimen based on microscopic examination of a $\mathrm{H} \& \mathrm{E}$ stained slide from the FFPE tissue, the accompanying pathology report, and additional information provided by the ordering physician.

\section{Comprehensive genomic Profiling}

CGP was performed on hybridization-captured, adaptor ligation-based libraries using DNA and/or RNA extracted from FFPE tumor in a Clinical Laboratory Improvement Amendments (CLIA)-certified and College of American Pathologists (CAP)-accredited laboratory (Foundation Medicine, Inc, Cambridge, Massachusetts, USA). The samples were sequenced for up to 406 cancer-related genes and select gene rearrangements. ${ }^{15}$ CD274 nonamplification SV mutations were defined as missense mutations, truncations, splice site mutations, and insertion/deletions, as previously described..$^{15}$ CD274 amplification was defined as ploidy +4 . Tumor mutational burden (TMB) was determined on up to $1.24 \mathrm{Mb}$ of sequenced DNA and TMB $\geq 10$ mutations/Mb (mut/Mb) was considered TMB-High per CDx approval. ${ }^{16}{ }^{17}$ Microsatellite instability (MSI) was performed from DNA sequencing up to 114 loci and MSI-High (MSI-H) was considered positive per CDx approval. ${ }^{18} 19$ In addition, as research use only (RUO), ultraviolet mutational signatures were called as described by Zehir et al. ${ }^{20}$

\section{Clonality, predicted germline versus predicted somatic mutation, and predicted missense functionality}

For the purposes of this study, a subclonal SV mutation was defined as a sample where $<50 \%$ of tumor cells were predicted to harbor the variant based on both the variant allele fraction and the pathologic and/or computational tumor cell purity estimates. A RUO somatic germline zygosity (SGZ) bioinformatics algorithm was used to determine whether mutations were likely somatic or germline, as previously described ${ }^{21}$ We assessed $C D 274$ missense mutation's functionality with several in silico methods including SIFT, MutationTaster, fathmm-MKL, and MetaSVM and recalibrated the scores to a rankscore so they can be compared with each other. ${ }^{22-25}$ The rankscore was on a scale of 0 to 1 with 0 being predicted to be a non-functional protein and 1 being predicted to be a functional protein.

\section{DAKO PD-L1 IHC 22C3 assay}

For a subset of cases, the PD-L1 DAKO 22C3 assay was run according to manufacturer instructions in a CLIAcertified and CAP-accredited laboratory (Foundation Medicine, Inc, Morrisville, North Carolina, USA).$^{26}$ The IHC cases were interpreted by board-certified pathologists specifically trained on the DAKO tumor proportion score (TPS) method, where tumor cell expression of PD-L1 was quantified. The DAKO TPS scoring method was defined as TPS=\# PD-L1 positive tumor cells/ (total \# of PD-L1 positive + PD-L1 negative tumor cells) ${ }^{27}$

\section{RESULTS \\ Landscape of CD274 SV mutations}

Overall, the frequency of $\mathrm{CD} 274 \mathrm{SV}$ mutations was low $(0.3 \%, 1081 / 314,631)$ in our cohort of 314,631 samples. A total of 577 unique variants were discovered; some mutations were recurrent, while others occurred only once in the entire cohort (table 1). Of the 1081 mutations, $49.9 \%(539 / 1081)$ were from metastatic specimens and $42.3 \%(457 / 1081)$ were from primary specimens. In $7.8 \%(85 / 1081)$ of cases it was unknown whether it was a primary or metastatic specimen. Of the 1081 samples with CD274 SV mutations, only $1.4 \%$ (15/1081) had cooccurring $\mathrm{CD} 274$ amplification.

The most common CD274 mutations were $\mathrm{R} 260 \mathrm{H}$ $(\mathrm{n}=51)$, R260C $(\mathrm{n}=18), \mathrm{R} 125 \mathrm{Q}(\mathrm{n}=12), \mathrm{C} 272 \mathrm{fs} * 13$ ( $\mathrm{n}=11)$, R86W ( $\mathrm{n}=10)$, and R113H ( $\mathrm{n}=10)$ (table 1, figure 1). $\mathrm{R} 260 \mathrm{C} / \mathrm{H}$ was the most frequent recurrent missense mutation; both substitutions have been observed in the germline of healthy subjects (gnomAD). Based on the SGZ algorithm, we found that $29.0 \%(20 / 69)$ of the codon R260 mutations were likely somatic and $55.1 \%$ $(38 / 69)$ were likely germline. The algorithm could not predict whether the variant was germline or somatic in $16.0 \%(11 / 69)$ of the samples. In addition, when we examined all the missense mutations $(\mathrm{n}=974)$, we saw that $51.0 \%(497 / 974)$ were predicted to be somatic, $24.7 \%$ (241/974) were predicted to be germline, and the algorithm could not predict whether the variant was germline or somatic in $24.2 \%(236 / 974)$ of the cases (online supplemental table 1). In addition, C272fs*13 is at an indel at a poly-A homopolymer, a sequence context that is highly mutable in the setting of MSI-H status. This mutation was significantly enriched in the MSI-H group $(0.01 \%, 5 / 5139)$ when compared with the nonMSI-H group $(0.002 \%, 6 / 309,492)$ (Fisher's exact test, $\mathrm{p}<0.0001$ ), suggesting that the variant is often a result of mismatch repair protein deficiency. This finding is reflected in the high CD274 SV mutation frequency in non-serous endometrial adenocarcinomas in this study.

The types of mutations in this cohort also varied, with missense mutations being the most common $(83.8 \%$, 


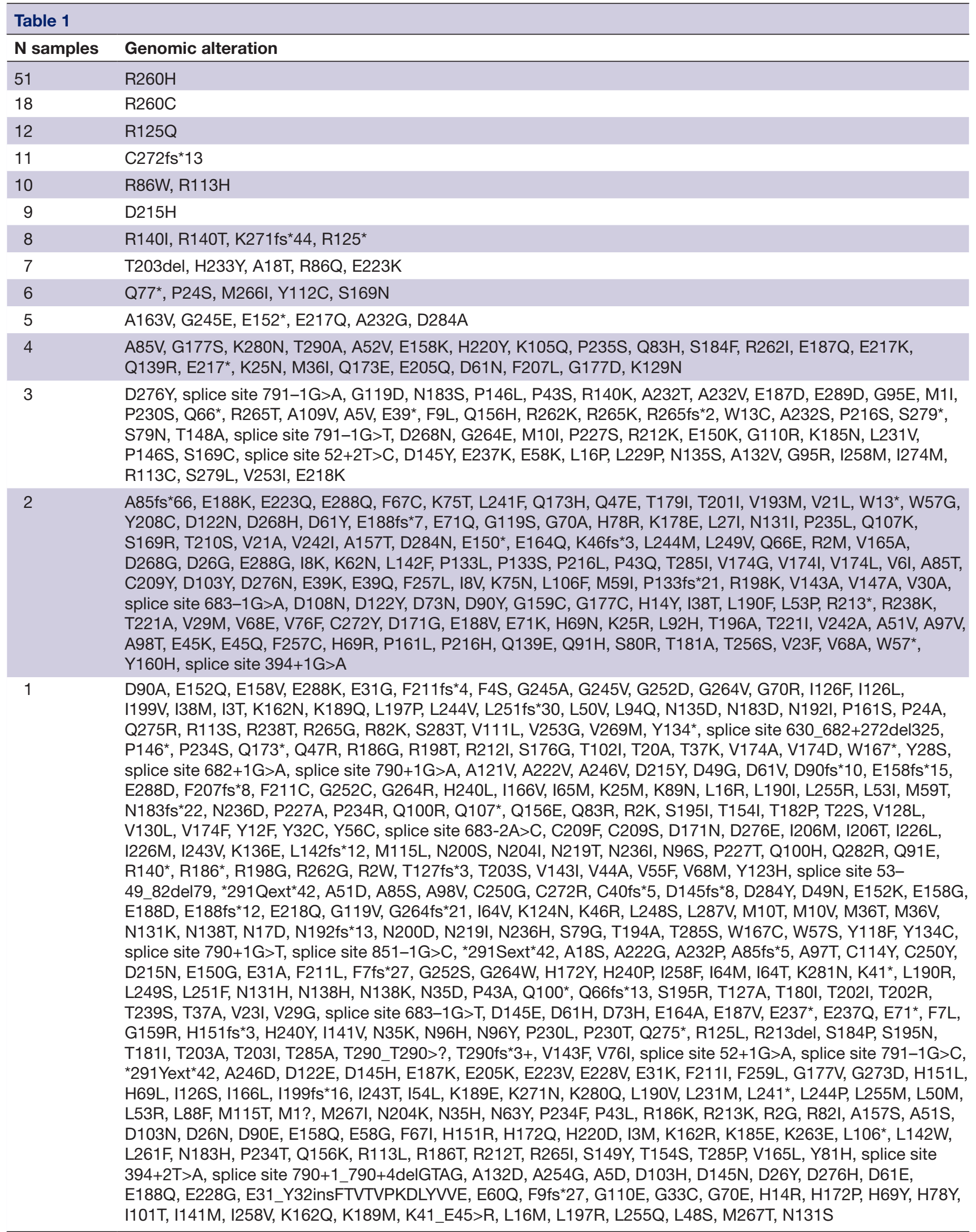




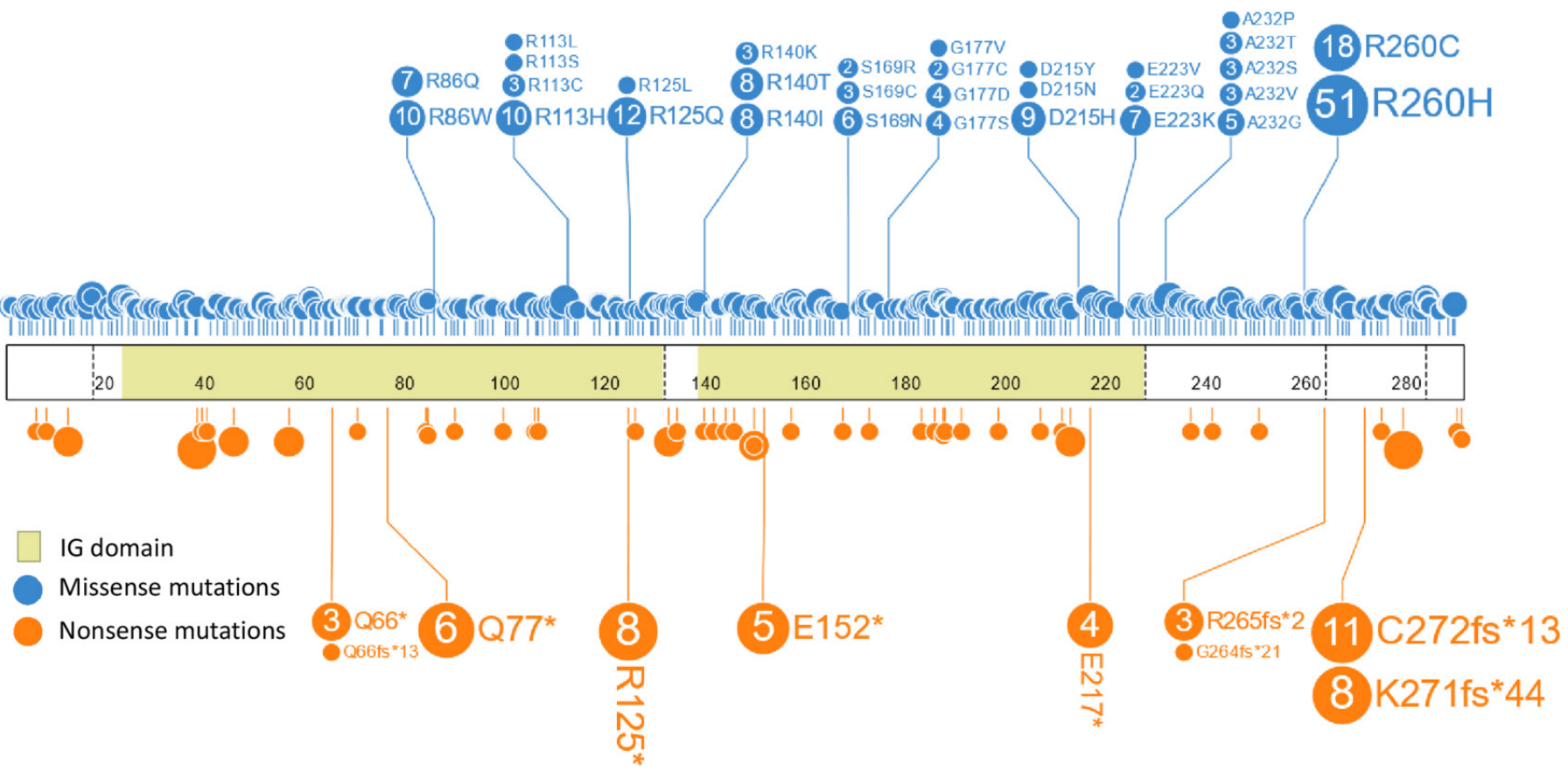

Figure 1 Lollipop plot of all the missense and nonsense mutations in the cohort. The most common CD274 mutations were R260H ( $n=51), \operatorname{R} 260 C(n=18), \operatorname{R} 125 Q(n=12)$, C272fs*13 $(n=11), R 86 W(n=10)$, and R113H $(n=10)$.

906/1081) and insertion/deletions being less common $(0.8 \%, 9 / 1081)$ (table 2). Multiple samples had complex CD274 mutations, defined as more than one CD274 genomic alteration observed in the sample. The most common type of complex $\mathrm{CD} 274$ mutation was alterations with two missense mutations $(1.9 \%, 21 / 1081)$, while other complex mutations consisted of a $\mathrm{CD} 274$ mutation with concurrent CD274 amplification $(1.4 \%, 15 / 1081)$ and/or rearrangement $(0.3 \%, 3 / 1081)$.

The prevalence of $C D 274$ mutations also varied depending on tumor type. The top five tumor types (minimum 800 total samples) with the highest rates of CD274 mutations in descending order were: diffuse large B-cell lymphoma $(1.9 \%, 19 / 997)$, cutaneous squamous cell carcinoma $(1.6 \%, 14 / 868)$, endometrial adenocarcinoma $(1.0 \%, 36 / 3740)$, unknown primary melanoma $(0.9 \%, 33 / 3679)$, and cutaneous melanoma $(0.8 \%$, $32 / 3874$ ) (figure 2, (online supplemental table 2). Interestingly, three of the five tumor types with the highest prevalence of $C D 274$ mutations usually occur on the skin. When we examined the mean TMB for these cases, we saw very high mean TMB and median TMB (cutaneous

Table 2

\begin{tabular}{lll}
\hline Total $(\mathbf{n = 1 0 8 1 )}$ & $\begin{array}{l}\text { Prevalence by mutation type } \\
(\mathbf{\%})\end{array}$ & Type of CD274 mutations \\
\hline Simple mutations & & Missense mutations \\
\hline 906 & 83.8 & Truncations \\
97 & 9.0 & Splice site mutations \\
\hline 24 & 2.2 & Insertion/deletions \\
\hline 9 & 0.8 & \\
\hline Complex mutations & & Two missense mutations \\
\hline 21 & 1.9 & Missense mutation with amplification \\
\hline 13 & 1.2 & Missense mutation with truncation \\
\hline 6 & 0.6 & S149Y, CD274-PLGRKT \\
\hline 1 & 0.1 & R140T, E187Q, deletion, CD274-CD274 \\
\hline 1 & 0.1 & L106*, amplification (copy number 90), CD274-CD274 \\
\hline 1 & 0.1 & P235S, S279L, amplification (copy number 12) \\
\hline 1 & 0.1 & R86Q, G95E, W57*, splice site 682+1G>A \\
\hline
\end{tabular}

\section{Prevalence by mutation type}

\section{Complex mutations}




\section{Prevalence of CD274 Muatations in Various Tumor Types}

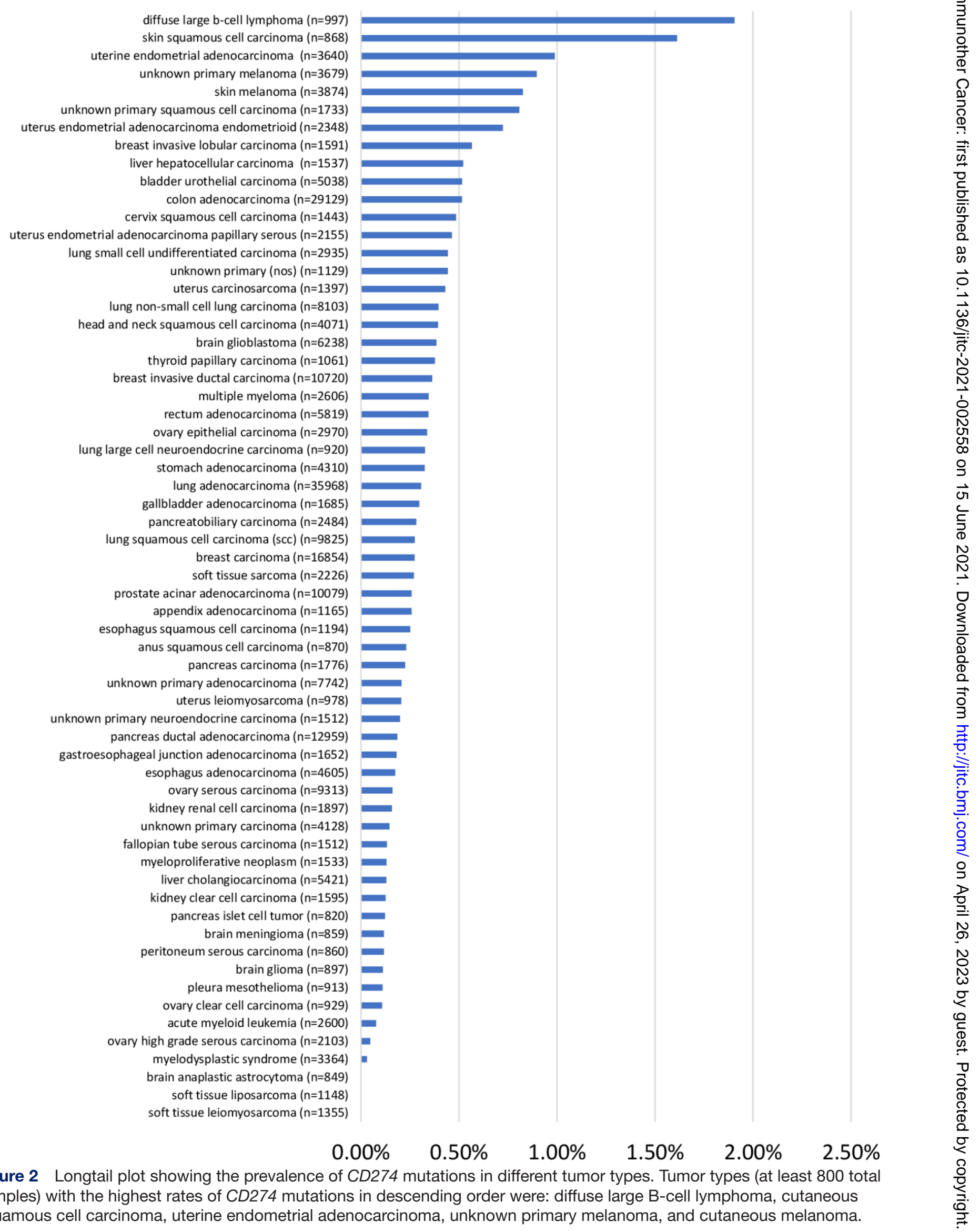


squamous cell carcinoma (151 mut/Mb, $100 \mathrm{mut} / \mathrm{Mb})$, cutaneous melanoma (133 mut/Mb, 126 mut/Mb), unknown primary melanoma (125 mut/Mb, 92 mut/Mb), respectively), suggesting that these were likely caused by ultraviolet exposure-induced hypermutation. This was further supported by the high prevalence of ultraviolet mutational signature in these tumor types (cutaneous squamous cell carcinoma $(84.6 \%, 11 / 13)$, cutaneous melanoma $(93.8 \%, 30 / 32)$, and unknown primary melanoma $(100 \%, 32 / 32)$. Whether these CD274 mutations are random 'passenger' mutations due to the high mutation rate should be further investigated.
Correlation of CD274 mutations with PD-L1 IHC tumor cell expression and predicted functionality models

Of the 1081 cases with CD274 mutations, $19.7 \%$ (213/1081) cases had PD-L1 IHC data; and of the 313,550 cases without CD274 mutations, $18.6 \%(58,218 / 313,550)$ cases had PD-L1 IHC data.

Most of the CD274 non-truncating mutations had low to no tumor-cell expression of PD-L1 (figure 3A, online supplemental table 3$)$. Of the $11 \mathrm{R} 260 \mathrm{H}$ cases concurrently tested with PD-L1 IHC, 81.8\% (9/11) had no PD-L1 expression and $2(18.2 \%)$ cases had low PD-L1 expression. Of the 5 E237K cases, $20 \%(1 / 5)$ had no PD-L1

A
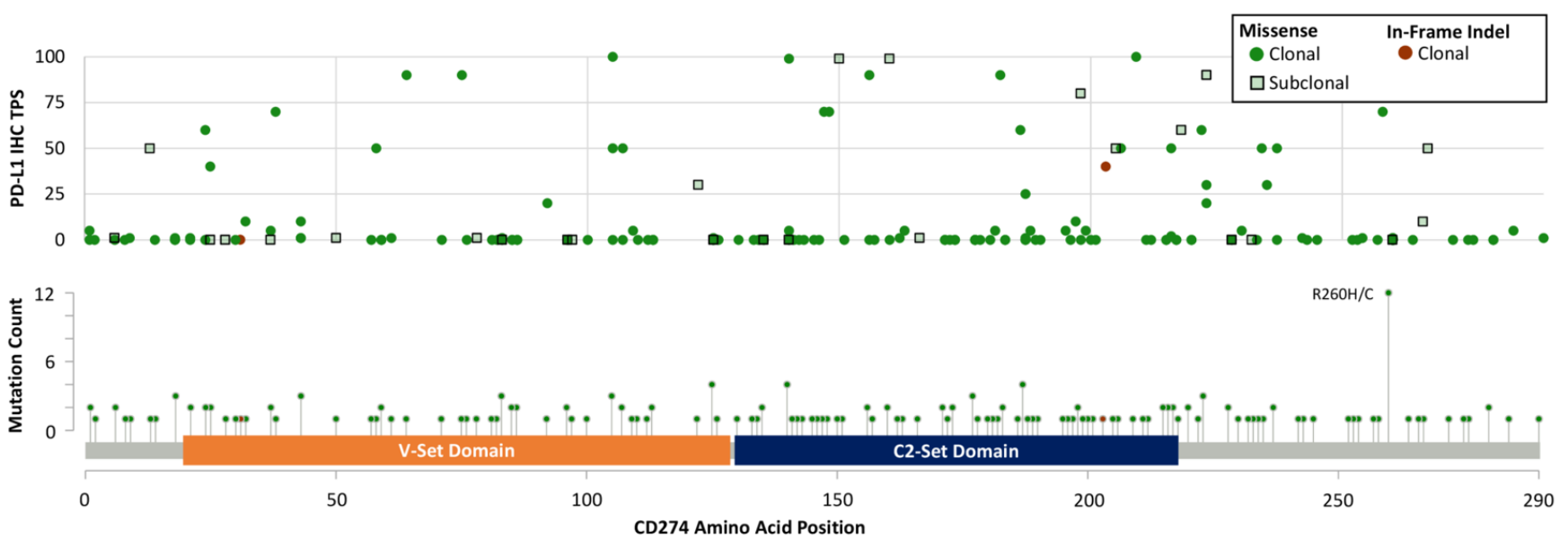

B
CD274 Missense Mutation Correlation with PD-L1 Protein Expression

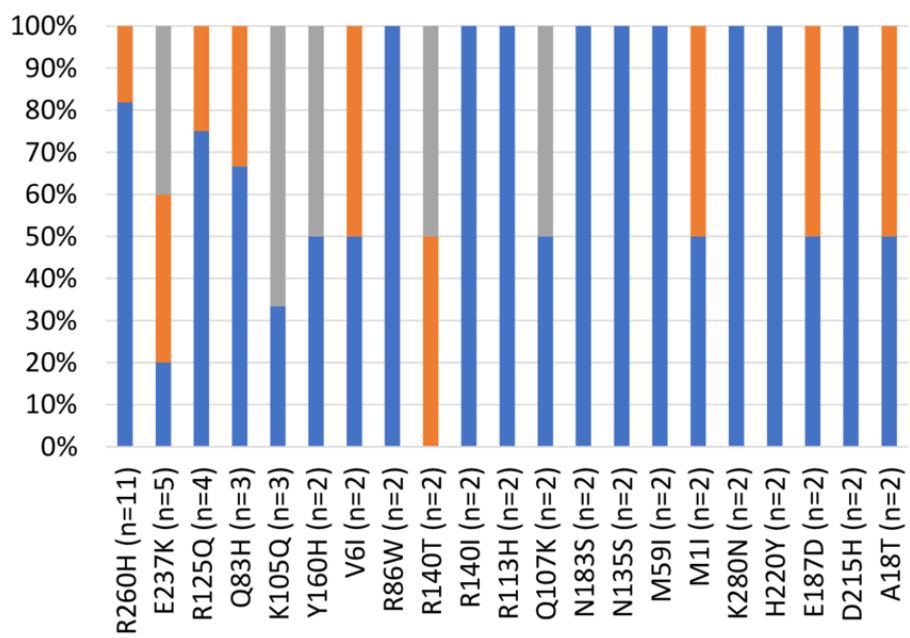

- TPS $=0$ (No Expression) =
C

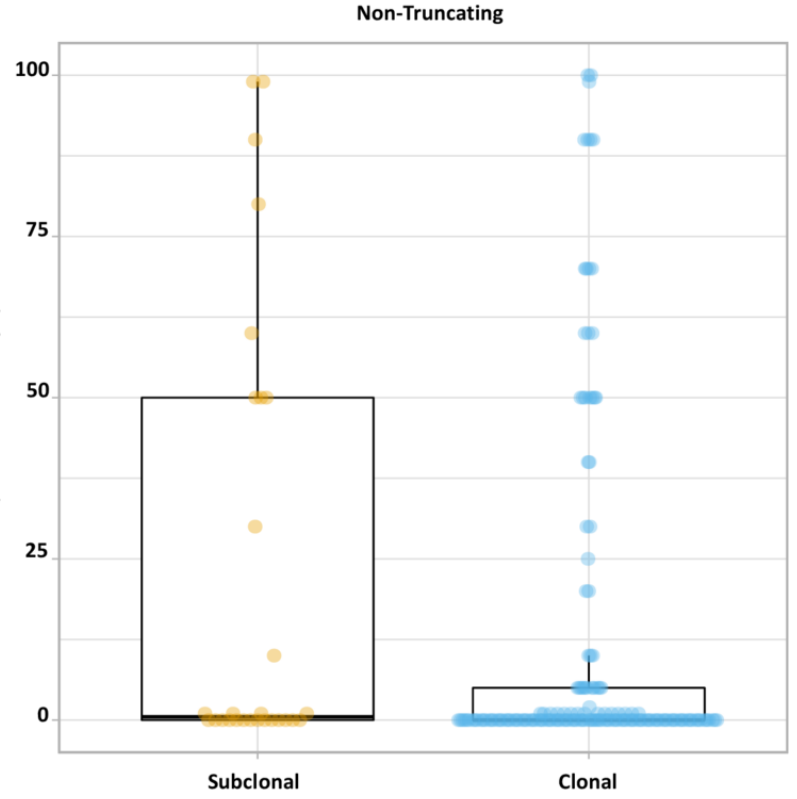

Figure 3 Correlation of CD274 non-truncating mutations with PD-L1 IHC tumor-cell expression. (A) Among non-truncating variants, 181 samples with missense substitutions and two in-frame indels were identified (lower). A subset of the variants was recurrent, with 12 samples harboring a substitution at R260. PD-L1 TPS scores corresponding to each sample with a nontruncating variant shown (upper). Subclonal variants are denoted with squares. (B) Correlation of $C D 274$ missense mutations and PD-L1 protein expression in mutations where at least two cases with PD-L1 IHC performed. (C) When examining the clonal $(n=153)$ versus subclonal $(n=28)$ missense mutations, we saw significantly lower PD-L1 IHC expression in the clonal missense mutation (mean: TPS $=11$ vs 22, respectively; ANOVA, $p=0.049$ ). Note: Subclonal variants were defined as samples where $<50 \%$ of tumor cells were predicted to harbor the variant based on the variant allele fraction and the pathologic and/or computational tumor-cell purity estimates. ANOVA, analysis of variance; IHC, immunohistochemistry; PD-L1, programmed cell death-ligand 1; TPS, tumor proportion score. 


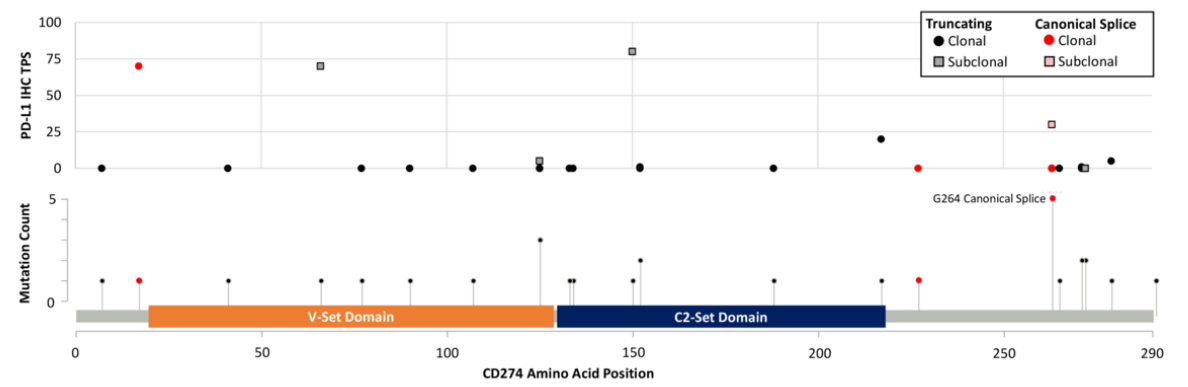

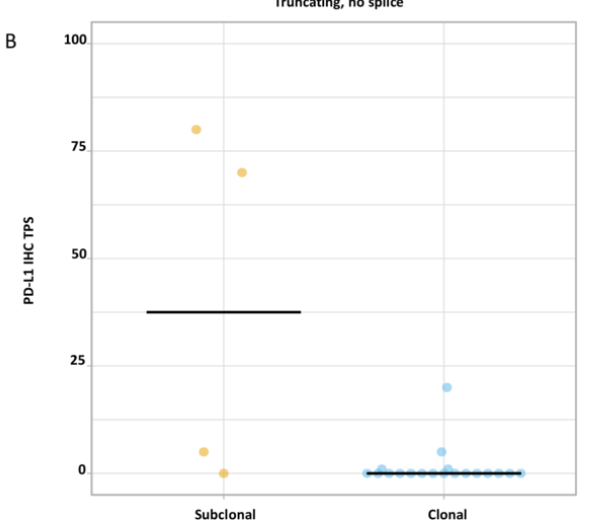

Figure 4 Correlation of CD274 truncating mutations with PD-L1 IHC tumor-cell expression. (A) Thirty-nine putative truncating variants were identified, including 12 nonsense mutations, 10 frameshift indels, and 7 canonical splice variants (lower). PD-L1 TPS scores corresponding to each sample with a non-truncating variant shown (upper). Subclonal variants are denoted with squares. (B) We saw a significantly lower level of PD-L1 expression in samples with a clonal truncating variant (nonsense or frameshift indel) when compared with samples with subclonal truncating variants (mean: TPS $=1$ vs TPS=38; ANOVA, $p<0.001$ ). Note: subclonal variants were defined as samples where $<50 \%$ of tumor cells were predicted to harbor the variant based on the variant allele fraction and the pathologic and/or computational tumor cell purity estimates. ANOVA, analysis of variance; IHC, immunohistochemistry; PD-L1, programmed cell death-ligand 1; TPS, tumor proportion score.

expression, $40 \%$ (2/5) had low PD-L1 expression, and $40 \%(2 / 5)$ had high PD-L1 expression (figure 3B). This difference in protein expression of the two mutations was significantly different (Fisher's exact test, $\mathrm{p}=0.036$ ). When we compared the PD-L1 protein expression of cases with $C D 274$ missense mutations $(\mathrm{n}=153)$ and cases without $C D 274$ mutations $(\mathrm{n}=58$ 218), we saw a lower level of PD-L1 IHC expression in the cases with CD274 mutations, though the difference was not statistically significant (mean: TPS=11 vs 13, respectively; analysis of variance (ANOVA), $\mathrm{p}=0.404$ ). When examining the clonal $(n=153)$ versus subclonal $(n=28)$ missense mutations, we saw significantly lower PD-L1 IHC expression in the clonal missense mutation (mean: TPS=11 vs 22, respectively; ANOVA, $\mathrm{p}=0.049$ ) (figure $3 \mathrm{C}$ ).

In addition, we examined the predicted functionality of each $C D 274$ missense mutation with multiple functionality prediction models including SIFT, MutationTaster, fathmm-MKL, and MetaSVM (online supplemental table 4). However, the predicted functionality did not have any significant correlation with PD-L1 IHC expression (online supplemental figure 1).

Thirty-nine putative truncating variants with concurrent PD-L1 IHC testing were identified, including 12 nonsense mutations, 10 frameshift indels, and 7 canonical splice variants (figure 4A, online supplemental table 2 ). When we compared the PD-L1 protein expression of cases with $C D 274$ clonal truncating variant (nonsense or frameshift indel) $(\mathrm{n}=18)$ and cases without $C D 274$ mutations $(n=58,218)$, we saw a lower level of PD-L1 expression in the cases with $C D 274$ mutations (mean: TPS $=1$ vs 13 , respectively; ANOVA, $\mathrm{p}=0.069$ ). In addition, we saw a significantly lower level of PD-L1 expression in samples with a clonal truncating variant (nonsense or frameshift indel) when compared with samples with subclonal truncating variants (mean: TPS=1 vs TPS=38; ANOVA, $\mathrm{p}<0.001$ ) (figure $4 \mathrm{~B}$ ).

\section{DISCUSSION}

This study describes a large cohort of 1081 clinically advanced malignancies with $C D 274$ non-amplification SV mutations, including 213 samples with concurrent PD-L1 protein expression levels. Similar to the COSMIC database $(0.4 \%, 229 / 51,849)$, the overall prevalence across tumor types was low $(0.3 \%, 1,081 / 314,631)$. Most of the SV mutations found were missense substitutions, with rarer nonsense and indel alterations. ${ }^{12}$ Last, the prevalence of CD274 SV mutations was higher in patients with MSI-H-associated endometrial and ultraviolet light exposed cutaneous cancers.

While most of the non-truncating SV CD274 mutations had low to no tumor-cell expression of PD-L1, the expression levels of PD-L1 differed among the various $C D 274$ SV mutation categories. Interestingly, of the $11 \mathrm{R} 260 \mathrm{H}$ cases concurrently tested with PD-L1 IHC, most showed little to no PD-L1 expression, suggesting that patients with $\mathrm{R} 260 \mathrm{H}$ mutations might not be treated with ICPI if only tested with PD-L1 IHC. The exact mechanism that causes the R260H to turn off PD-L1 expression and/or to potentially interfere with the binding of ICPI to PD-L1 ligand should be further studied, especially given the relatively high prevalence of $\mathrm{R} 260 \mathrm{H}$ mutation in our cohort $(4.7 \%$, 51/1081). This contrasted with the five E237K cases, where most cases had some PD-L1 expression, suggesting that the E237K has little to no effect on PD-L1 protein expression. Most other variants were only observed in a single sample (with or without concurrent PD-L1 expression data) due the rarity of the mutations. This rarity of individual variants, even in a very large database of 
cases with CGP, makes the clinical significance of these uncommon mutations less clear. However, as more studies evaluate the PD-L1 protein expression of tumors with $C D 274$ SV mutations, these data can be aggregated to better understand the functionality of $C D 274$ mutations on PD-L1 protein expression.

As expected, samples with a clonal truncating mutation had a lower level of PD-L1 expression when compared with cases without a $C D 274$ mutation and to samples with a subclonal truncating mutation. These data suggest that when a clonal truncating event is observed in $\mathrm{CD} 274$, PD-L1 expression is inhibited, but in the setting of a subclonal truncation, clinical assessment of sample-level PD-L1 expression is often not affected. We hypothesize that clonal truncating variants can potentially act as resistance biomarkers for ICPI due the lack of PD-L1 protein present on the tumor cells as exemplified by our PD-L1 IHC expression data. With decreased/no ligand for the PD-L1/PD-1 inhibitors to bind to, the efficacy of ICPI would likely be diminished.

In terms of CD274 missense mutations, these could mediate resistance to ICPI due to potential steric or affinity-altering interferences in the binding of the PD-L1 ligand to the PD-1 receptor, similar to a resistance mechanism described for ROS1, though further studies are needed to evaluate this hypothesis. ${ }^{28}{ }^{29}$ In our cohort of cases with CD274 missense mutations, we observed a slightly lower level of PD-L1 IHC staining in the cases with CD274 missense mutations when compared with cases without $C D 274$ mutations, and we also saw significantly lower PD-L1 IHC staining in the clonal missense mutation when compared with the subclonal missense mutations. We hypothesize that this is likely due to the lower rates of PD-L1 antibody binding (from the IHC assay) to the PD-L1 ligand on the tumor cells instead of actual lower PD-L1 protein expression. In addition, we saw no correlation between the predicted functional status of these missense mutations and PD-L1 IHC expression since these prediction algorithms do not formally consider the steric or binding affinity interferences that can result from missense mutations into the functionality assessment. Taken together, we have some preliminary data that support our hypothesis of the potential mechanism of resistance of tumors with CD274 missense mutations to ICPI, and this should be further investigated in both in vitro and clinical studies.

This study has two primary limitations. First, we only have PD-L1 IHC on a limited subset of the samples with CD274 mutations. The second limitation of this study is that we do not have any clinical outcome data of patients with $C D 274$ mutations and treated ICPI to assess the clinical impact of ICPI on patients with $C D 274$ mutations.

\section{CONCLUSIONS}

We presented the landscape of $C D 274$ mutations in a large pan-cancer cohort that can be used as a reference for examining $\mathrm{CD} 274$ mutations as potential resistance biomarkers for ICPI. Furthermore, we presented novel data on the correlation of $\mathrm{CD} 274$ mutations and PD-L1 protein expression, providing important data on the potential functionality of these mutations that will serve as a basis for future research.

Contributors RSPH, BD and JSR were invovled in study conception/design. Provision of study material or patients were done by RSPH, BD, KM, MH, DAM, SR and JSR. Collection and/or assembly of data were performed by RH and BD. Data analysis and interpretation and manuscript writing were done by all the authors. All authors gave final approval of manuscript.

Funding The authors have not declared a specific grant for this research from any funding agency in the public, commercial or not-for-profit sectors.

Competing interests All authors of the manuscript are employees of Foundation Medicine, Inc, which is a wholly owned subsidiary of Roche and receives stock from Roche.

Patient consent for publication Not required.

Ethics approval Approval for this study was obtained from the Western Institutional Review Board Protocol No 20152817.

Provenance and peer review Not commissioned; externally peer reviewed.

Data availability statement All data relevant to the study are included in the article or uploaded as supplementary information. The data generated by the research that support our article will be provided in the supplements.

Supplemental material This content has been supplied by the author(s). It has not been vetted by BMJ Publishing Group Limited (BMJ) and may not have been peer-reviewed. Any opinions or recommendations discussed are solely those of the author(s) and are not endorsed by BMJ. BMJ disclaims all liability and responsibility arising from any reliance placed on the content. Where the content includes any translated material, BMJ does not warrant the accuracy and reliability of the translations (including but not limited to local regulations, clinical guidelines, terminology, drug names and drug dosages), and is not responsible for any error and/or omissions arising from translation and adaptation or otherwise.

Open access This is an open access article distributed in accordance with the Creative Commons Attribution Non Commercial (CC BY-NC 4.0) license, which permits others to distribute, remix, adapt, build upon this work non-commercially, and license their derivative works on different terms, provided the original work is properly cited, appropriate credit is given, any changes made indicated, and the use is non-commercial. See http://creativecommons.org/licenses/by-nc/4.0/.

\section{ORCID iD}

Richard S.P. Huang http://orcid.org/0000-0001-8395-5168

\section{REFERENCES}

1 Li Y, Li F, Jiang F, et al. A Mini-Review for Cancer Immunotherapy: Molecular Understanding of PD-1/PD-L1 Pathway \& Translational Blockade of Immune Checkpoints. Int J Mol Sci 2016;17. doi:10.3390/ijms17071151. [Epub ahead of print: 18 Jul 2016].

2 Schachter J, Ribas A, Long GV, et al. Pembrolizumab versus ipilimumab for advanced melanoma: final overall survival results of a multicentre, randomised, open-label phase 3 study (KEYNOTE-006). Lancet 2017;390:1853-62.

3 Pai-Scherf L, Blumenthal GM, Li H, et al. Fda approval summary: pembrolizumab for treatment of metastatic non-small cell lung cancer: first-line therapy and beyond. Oncologist 2017;22:1392-9.

4 Schmid P, Adams S, Rugo HS, et al. Atezolizumab and nabpaclitaxel in advanced triple-negative breast cancer. $N$ Engl J Med 2018;379:2108-21.

5 FDA. List of Cleared or Approved Companion Diagnostic Devices (In Vitro and Imaging Tools). [Available from. Available: https://www. fda.gov/medical-devices/vitro-diagnostics/list-cleared-or-approvedcompanion-diagnostic-devices-vitro-and-imaging-tools

6 Gjoerup O, Brown CA, Ross JS, et al. Identification and utilization of biomarkers to predict response to immune checkpoint inhibitors. Aaps J 2020;22:132.

7 Chung HC, Ros W, Delord J-P, et al. Efficacy and safety of pembrolizumab in previously treated advanced cervical cancer: results from the phase II KEYNOTE-158 study. J Clin Oncol 2019;37:1470-8. 
8 Hellmann MD, Paz-Ares L, Bernabe Caro R, et al. Nivolumab plus ipilimumab in advanced non-small-cell lung cancer. $N$ Engl J Med 2019;381:2020-31.

9 Fabrizio FP, Trombetta D, Rossi A, et al. Gene code CD274/PD-L1: from molecular basis toward cancer immunotherapy. Ther Adv Med Oncol 2018;10:1758835918815598.

10 NCBI. Matched annotation from NCBI and EMBL-EBI (MANE)[. Available: https://www.ncbi.nlm.nih.gov/refseq/MANE/2020

11 Tate JG, Bamford S, Jubb HC, et al. Cosmic: the Catalogue of somatic mutations in cancer. Nucleic Acids Res 2019;47:D941-7.

12 Cosmic. cosmic v92, released 27-AUG-20 cancer.sanger.ac.uk2020.

13 Huang RSP, Haberberger J, Severson E, et al. A pan-cancer analysis of PD-L1 immunohistochemistry and gene amplification, tumor mutation burden and microsatellite instability in 48,782 cases. Mod Pathol 2021:34:252-63.

14 O'Malley DP, Yang Y, Boisot S, et al. Immunohistochemical detection of PD-L1 among diverse human neoplasms in a reference laboratory: observations based upon 62,896 cases. Mod Pathol 2019;32:929-42.

15 Frampton GM, Fichtenholtz A, Otto GA, et al. Development and validation of a clinical cancer genomic profiling test based on massively parallel DNA sequencing. Nat Biotechnol 2013;31:1023-31.

16 FDA. Fda approves pembrolizumab for adults and children with TMB-H solid tumors. Available: https://www.fda.gov/drugs/drugapprovals-and-databases/fda-approves-pembrolizumab-adults-andchildren-tmb-h-solid-tumors 2020

17 Chalmers ZR, Connelly CF, Fabrizio D, et al. Analysis of 100,000 human cancer genomes reveals the landscape of tumor mutational burden. Genome Med 2017;9:34.

18 FDA. Fda grants accelerated approval to pembrolizumab for first tissue/site agnostic indication. Available: https://www.fda.gov/drugs/ resources-information-approved-drugs/fda-grants-acceleratedapproval-pembrolizumab-first-tissuesite-agnostic-indication [Accessed on 01/30/2020].
19 Trabucco SE, Gowen K, Maund SL, et al. A novel next-generation sequencing approach to detecting microsatellite instability and PanTumor characterization of 1000 microsatellite Instability-High cases in 67,000 patient samples. J Mol Diagn 2019;21:1053-66.

20 Zehir A, Benayed R, Shah RH, et al. Mutational landscape of metastatic cancer revealed from prospective clinical sequencing of 10,000 patients. Nat Med 2017;23:703-13.

21 Sun JX, He Y, Sanford E, et al. A computational approach to distinguish somatic vs. germline origin of genomic alterations from deep sequencing of cancer specimens without a matched normal. PLoS Comput Biol 2018;14:e1005965.

22 Kim S, Jhong J-H, Lee J, et al. Meta-Analytic support vector machine for integrating multiple omics data. BioData Min 2017;10:2.

$23 \mathrm{Ng}$ PC, Henikoff S. SIFT: predicting amino acid changes that affect protein function. Nucleic Acids Res 2003;31:3812-4.

24 Shihab HA, Gough J, Cooper DN, et al. Predicting the functional consequences of cancer-associated amino acid substitutions. Bioinformatics 2013;29:1504-10.

25 Schwarz JM, Cooper DN, Schuelke M, et al. MutationTaster2: mutation prediction for the deep-sequencing age. Nat Methods 2014;11:361-2

26 DAKO. Pd-L1 IHC 22C3 pharmDx package insert. Available: https:// www.accessdata.fda.gov/cdrh_docs/pdf15/P150013c.pdf2020

27 DAKO. Pd-L1 IHC 22C3 pharmDx interpretation manual - NSCLC. Available: https://www.agilent.com/cs/library/usermanuals/public/ 29158_pd-I1-ihc-22C3-pharmdx-nsclc-interpretation-manual.pdf [Accessed on 5/9/2019].

28 Huang RSP, Gottberg-Williams A, Vang P, et al. Correlating ROS1 protein expression with ROS1 fusions, amplifications, and mutations. JTO Clinical and Research Reports 2020;100100.

29 Huang RSP, Haberberger J, Sokol E, et al. Clinicopathologic, genomic and protein expression characterization of 356 ROS1 fusion driven solid tumors cases. Int J Cancer 2021;148:1778-88. 